\title{
Studies of Voluntary Visual Attention- Theory, Methods, and Psychometric Issues
}

\author{
Jum C. Nunnally \\ Vanderbilt University \\ L. Charles Lemond \\ Southwestern at Memphis \\ William H. Wilson \\ Middle Tennessee State University
}

\begin{abstract}
The paper discusses the study of voluntary visual attention (VVA), a relatively new area of active experimentation. VVA concerns "natural" viewing behavior or visual browsing when the subject is under no constraints regarding the distribution of attention. This is contrasted with traditional studies of directed visual attention, such as the typical study of visual judgment in tachistoscopic research. Discussed are (1) the logic of investigating VVA, (2) a comprehensive set of constructs that are thought to be of theoretical importance, (3) methods for calibrating these variables in terms of treatment parameters, (4) the logic of scaling both independent and dependent variables, (5) a summary of salient findings, (6) some recent findings not previously reported, and (7) an overview of the psychometric issues in the study of VVA.
\end{abstract}

Traditionally, most investigations of vision have concerned directed attention, in which the subject is instructed to look at a target object and make a judgmental response of some kind. Studies of visual reaction time to various types of words, of effects of stimulus intensity on binocular rivalry, and of perceived visual motion are some examples. In these and other traditional studies of directed attention, the subject is usually told where to look, what to look for, and how

APPLIED PSYCHOLOGICAL MEASUREMENT

Vol. 1. No. 2 Spring 1977 pp. 203-218

(C) Copyright 1977 West Publishing Co. to respond. Allied with this important tradition of studies of directed attention, a new sub-area of research has developed-that of voluntary visual attention (VVA).

One type of VVA experiment concerns the amount of time spent looking at different objects within a visual field vhen there are no instructions regarding how attention should be distributed. In other approaches, subjects view displays one at a time, and are allowed to control freely the time looking at each. In essence, VVA concerns "visual browsing", or the "natural" distribution of attention when the subject is not under instructions to look at particular objects, to make judgments about them, or to distribute his or her attention in any other prescribed way.

Studies of VVA are part of an emerging interest in exploratory behavior that has developed over the last 20 years and has been evidenced by a moderate amount of research. Nunnally and Lemond (1973) summarized the place of VVA in an overall model concerning the components of exploratory behavior and presented a theoretical scheme for VVA.

A very simple example from one part of a major investigation (Wilson, 1973) will serve to illustrate a typical study of VVA. The study concerned the effect of the homogeneity versus 
heterogeneity of objects. In the most homogeneous displays, ten copies of the same face appeared. In the heterogeneous display, ten different faces appeared. Essentially the subject was allowed to look at each slide as long as he elected, then push a button to move to the next slide. The dependent variable was amount of time in seconds spent viewing each picture. As was expected, heterogeneous displays were viewed longer than homogeneous displays. More complex equipment, procedures, and visual displays are employed in the studies of VVA, but in all cases the subject controls the distribution and duration of attention.

Numerous psychometric issues have been encountered in our studies of VVA. Many of our independent variables concern characteristics of the visual stimuli, such as the effect of novelty on VVA. In earlier studies, simple comparisons were made of drawings of a novel object with drawings of a banal counterpart, e.g., a distorted shape of a cow as compared to an ordinary cow. In order to more fully understand the effects of novelty and other stimulus characteristics on VVA, scales were developed to measure degrees of the stimulus characteristic in question. For example, we developed scales to measure novelty at five different levels and scales to measure physical complexity at many different levels. The development of such scales involved numerous psychometric issues and experiments on scaling. This paper will (1) introduce readers who are unfamiliar with the topic to the study of VVA, (2) summarize some theoretical points of view which represent the most recent version of the authors' thinking in that regard, (3) describe a variety of psychometric issues that arise in a research program of this kind, and (4) summarize some major psychometric evidence regarding research in this area.

\section{Major Theoretical Constructs}

By now there are numerous theoretical positions regarding the major determinants of voluntary visual attention, among which are Berlyne (1960, 1966), Dember and Earl (1957),
Fiske and Maddi (1961), Fowler (1965), and Nunnally and Lemond (1973). The senior author and his colleagues began their investigations about ten years ago based on a loosely conceived cognitive point of view, which emphasized the encoding of the visual stimulus as being primarily important in determining amount of VVA. By the term "encoding" are meant all of the subprocesses involved in making sense out of a stimulus-detecting, identifying, recognizing, naming, and associating the visual stimulus with other stimuli "in the mind" of the viewer. Broadly speaking, this is referred to as a meaning-processing point of view, which concerns the activity of subjects in attributing meaning to the stimulus regardless of its characteristics.

This essentially cognitive point of view has been opposed to a motivational point of view in a variety of experiments. The motivational point of view emphasizes specific emotional states such as arousal or tedium. Both the cognitive and motivational points of view have much to contribute. But a rapproachment is required if VVA is to be explored productively. The following represents an abbreviated version of our present point of view concerning the major $\operatorname{cog}$ nitive and motivational constructs relating to VVA.

\section{Cognitive Variables}

Information conflict. Information conflict refers to competing cues for encoding, as we defined encoding above. An example is a drawing of a cow with the trunk of an elephant. To a lesser extent, information conflict occurs at any place in daily life where a visual stimulus, such as a horse standing in the lobby of a hotel, simply does not "fit" into the scheme of what we already know or are accustomed to seeing. It is our hypothesis that information conflict is a powerful determinant of VVA and usually will dominate any other variable that is present. Discussions of this point of view are given in Nunnally (1972) and Nunnally and Lemond (1973).

Number of representational elements. A second cognitive variable concerns the number 
of thing-like objects that can be recognized in a visual display. In studies of random polygons, it became apparent that people were seeing all kinds of things in what had been intended to be random jumbles of lines that varied only in terms of sheer physical complexity. In various psychophysical scalings of these geometrical forms, we obtained association hierarchies, naming responses, and ratings of the number of things that could be seen. Highly reliable differences were found among the stimuli in number of things seen, and this variable correlated highly with the complexity of the stimuli as defined in information-theoretic terms. In randomly constructed geometrical forms and in some other materials used to study complexity (e.g., dot patterns), it is best to speak of the thing-like elements as quasi-representational, because they are much like the elements that can be seen in ink blot tests-that is, portions of the figures remind people of things (e.g., a bird) rather than being directly representative of them. Frequently these quasi-representational elements in visual displays have some information conflict, which according to our hypotheses would further enhance amount of VVA.

Complexity of physical attributes. In much of our theorizing, the major emphasis was placed on the meaningful components of the visual stimulus as evidenced in the two variables mentioned above, and the purely physical characteristics of the stimuli were de-emphasized. This is in contrast to some other authors who rely heavily on the purely informationtheoretic properties of the stimuli rather than on meaning-processing. However, it became apparent that there are some purely physical dimensions of stimuli that are determinants of VVA. An example is in our extensive collection of geometrical forms, where it was found that some variance in VVA could be explained by sheer complexity that could not be explained by our other cognitive variables. By sheer complexity we mean physical complexity one can measure in terms of length, angles, and number of parts, and can thus be expressed in informationtheoretic terms.
Other physical attributes. We recognize the possibility that there are other purely physical attributes of stimuli that may be important in determining VVA. Such variables are symmetry, distribution of detail in the stimulus configuration, contrast within the stimulus, and others. For example, VVA may be elicited by sharp bor-. ders in terms of changes from black to white or from one color to another.

Associations-cognitive. Part of our overall concept of meaning-processing is that amount of VVA depends upon the associates the individual has to the stimulus. Visual displays can be investigated by the typical method of free association, and association hierarchies can be obtained. It is hypothesized that information conflict is present in any display where there are two or more strongly competing associates, an hypothesis which proved subsequently to be the case. However, there are probably many other cognitive aspects of association that are important. For one example, the number of definite associates should act like a number of representational elements to hold VVA. Also, the nature of these associates should be important; for example, the associates themselves might vary in difficulty of encoding. This category is specifically labeled "cognitive" because later we want to mention the effects of affectual associations on VVA.

Signal characteristics-cogniiive. The term. signal is being used in its traditional sense to refer to the information that an object supplies about other objects or events. The primary examples are words, e.g., as on stop signs, billboards, store fronts, and other places. Nonverbal signals are such as stone or wood markers used by primitive hunters to signal directions, distances, presence of game or dangers that lay ahead. Berlyne (1971) presents an extensive discussion of the place of such signals in exploratory behavior generally, which includes VVA. Here we distinguish between the cognitive components of such signals and the motivational components (discussed below). We hypothesize that any object with signal characteristics draws and holds VVA. For example, it seems that people are compulsively drawn to read almost 
any visual material that comes before their eyes, notice arrows indicating one-way streets, or gaze at the movements of people that signal some type of activity (e.g., many people crowding into the door of a store). We regard these cognitive aspects of signals as another part of the overall meaning-processing point of view about VVA.

\section{Motivational Variables}

The major motivational variables that are considered in our overall conceptual scheme are as follows.

Arousal. The construct of arousal (or activation) is usually indexed in terms of physiological variables such as electrical activity of the brain, heart rate, cortico-steriod levels in the blood, GSR, and pupillary response. Berlyne (1971) has based a theory of exploratory behavior around the concept of arousal. Essentially the major proposition relating to VVA is that there are optimum levels of arousal produced by visual configurations which enhance amount of attention. This optimum level depends upon the maturity of the organism, various types of individual differences, organic states at the moment, and numerous characteristics of the stimulus itself. Similar positions have been advocated by Fiske and Maddi (1961), Dember and Earl (1957), and others. All of these points of view are discussed well by Berlyne $(1971,1974)$ in relation to aesthetics.

Specific emotions. It is hypothesized that the emotions evoked by visual stimuli are important determinants of amount and kinds of VVA. For example, in addition to the other variables that influence VVA, it probably makes a difference whether the visual configuration induces anger, joy, disgust, sadness, surprise, and other emotions. Effects of such specific emotions on VVA have not been investigated to date, but will be in the future.

Tedium. Various authors have emphasized the influence of tedium or boredom in relation to VVA (e.g., Cantor and Cantor, 1966, and Fowler, 1965). It would be more proper to say that tedium potentially explains why a stimulus is not afforded much VVA. In its simplest form, tedium is expressed as a direct function of exposure time to a particular stimulus. So the theory goes, tedium becomes conditioned to the stimulus and obeys laws of classical conditioning as regards repeated exposures, intertrial intervals, and extinction over a period of non-reinstatements of tedium. Although earlier studies attempted to explain away any effects of tedium on VVA (e.g., Faw and Nunnally, 1970, 1971), in subsequent investigations (e.g., Lemond, 1973) it gradually became apparent that the concept of tedium might be important, particularly in studies of prior familiarization of stimuli.

Signal characteristics - motivational. As mentioned, some of the variables that have cognitive implications can also have definite motivational implications. By motivational aspects of signals we mean information that will help the individual safeguard himself from unpleasant events and/or lead him to desired outcomes. The sign on the highway that says "Gas and Restaurant Two Miles Ahead" provides information of potential motivational importance. Nonverbal signals, such as cars backed up on the road ahead or physical signs of ill-health, can also have motivational implications. We certainly hypothesize that these motivational implications of signals are important as determinants of VVA.

Associations-motivational. In addition to their cognitive implications, the motivational implications of visual displays potentially are important in VVA. For example, in a painting not only is there information to process, numbers of objects to view, arousal, and the various other determinants of attention, but also what is depicted in the display frequently has motivationally significant associations. There may be reminders of events in childhood, a face resembling an important person in one's life, or an object that brings old memories that induce strong emotions of one type or another. It is probably the case that many of these motivation-related associations are at low levels of consciousness, such as cues that induce feelings relating to sex, disgust, or personal agrandizement. This is a difficult category in which to perform operation- 
alized research. But such motivationally-relevant associations potentially are important in certain aspects of VVA, particularly in aesthetic preferences.

\section{Measurement of Dependent Variables in Studies of VVA}

VVA refers to any behavior which results in a stimulus being fixated on, brought into view, maintained in view, or voluntarily brought back into view after it is gone. Although the methods of investigation differ in terms of formal operations, all methods present the subject with one or more visual configurations and, by one method or another, allow him to view the stimuli for varying amounts of time. The major difference among methods is that in some cases the individual is shown a number of different visual configurations at the same time on a screen, whereas in others he is shown visual configurations serially one at a time. Visual congigurations include randomly constructed geometrical forms, patterns of dots, drawings of novel animal-like things, drawings of furniture or other objects, and pictures of real-life objects and social scenes. The major methods employed in studies of VVA are as follows:

Gross head movements. Methods for the study of gross movements of the head involve simultaneously exposing subjects to several stimuli, and forcing the subject to make a head movement in order to focus on a stimulus. For example, Faw and Nunnally (1968b) presented stimuli on two viewing screens separated by about three feet, and each child was seated in a chair facing the screens. This forced the child to make a gross head movement in order to bring either of the two stimuli into view. Looking behavior was monitored through a one-way looking screen from a separate room. The amount of time spent viewing each stimulus in each pair provided an index of VVA. The primary advantage of this response measure is that it can be employed in semi-naturalistic settings which make viewing "natural."

Visual fixations. A popular method of meas- uring visual fixations has been employed by Berlyne (1958) and Nunnally and his associates (e.g., Faw and Nunnally, 1967). The details vary, but essentially a small number of stimuli are presented at a short distance $($ e.g., $60 \mathrm{~cm}$.) from the subject's face. A chin rest or other device is used to prevent gross head movements. The stimuli are separated by enough distance to require the subject to make gross eye movements in order to view any particular stimulus. Visual fixations are either judged by a hidden rater (e.g., Berlync, 1958), photographed (e.g., Faw and Nunnally, 1967), or video-taped (e.g., Lemond, 1973).

The primary advantage of studying visual fixations is that the procedure is rather simple and requires little understanding or physical effort from the subject. Lemond (1973) reported an inter-judge agreement of $97 \%$ on the scoring of video-taped records of the visual fixations of adults. Another advantage is that it is very easy to disguise the purpose of the investigation in such a manner that the subject is unaware that the amount of time spent looking at different stimuli is important. In post-experimental testing, it has been found that subjects are unaware of which parts of the visual display they viewed longer (e.g., Durham, Nunnally, and Lemond, 1971).

Instrumental viewing responses. A third approach to the measurement of VVA concerns instrumental responses made by the subject to bring a stimulus into view or to keep a stimulus in view. This includes such responses as button pushing, bar pressing, and lever pulling. Many different types of apparatus have been employed for this purpose. For example, Berlyne (1957) used a tachistoscope to present visual stimuli to subjects. When the subject pressed a key, a stimulus became visible for .14 second. Subjects were allowed to view each stimulus as many times as they wished. Various types of "looking boxes" have also been employed, in which the subject pushes buttons to light up different screens on a box. Each screen illuminates a different visual configuration (e.g., Nunnally, Duchnowski, and Knott, 1967). The data of in- 
terest using these instrumental viewing procedures are the number of times or amount of time the subject looks at each stimulus.

By far the most popular procedure for studying instrumental viewing responses has been that of "free looking time" (e.g., Leckart and Bakan, 1965). With this procedure, subjects are allowed to view a series of stimuli one at a time through the use of a remote-control slide projector. A stimulus remains in view until the subject pushes a button which advances the projector to the next stimulus. An example is in studying viewing time as a function of different levels of complexity of geometric forms. Sixty slides, each containing a geometric form at a particular level of complexity are randomly ordered in a tray of slide projector. Subjects are shown how to operate the remote-control switch and are left to view the stimuli, freely distributing their viewing time. The data of interest are amount of time spent viewing each stimulus.

The primary advantages of studying free looking time over other types of responses is that it is very simple, economical, and allows one to gather large amounts of data in rather short periods of time. A potential disadvantage of investigating free looking time is that the task may appear rather trivial to adults. Also, children may get lost in the sheer fun of manipulating the projector, to the detriment of any findings regarding visual investigation. Neither of these has appeared to be a major disadvantage in our investigations so far.

\section{Absolute and Comparative Measures}

An important distinction is between absolute and comparative viewing time. The use of a free looking time procedure is a cardinal example of the former. Although there are subtie constraints on time spent viewing the stimuli, the subject is under the impression that he can spend as much time as he likes viewing each stimulus. Thus, the stimuli are not obviously in competition with one another as regards amount of looking time. Several other methods involving instrumental viewing responses are measures of absolute looking time. In contrast, gross head movements, visual fixations, and some intrumental viewing responses obviously involve comparative looking time. This is the case in any situation where the individual is shown two or more stimuli at the same time and the response measure concerns the percentage of the time spent looking at one stimulus rather than others. One might expect that there would be important differences in VVA in these two conditions, but no important differences have been found to date (see Nunnally and Lemond, 1973).

Instructions. The instructions given the subject have been shown to play an important role in determining visual investigation (see Nunnally and Lemond, 1973). For example, data frequently are different if instructions emphasize pleasantness of the stimuli rather than remembering or otherwise encoding the stimuli. In spite of the proven importance of instructional sets, the specific instructions frequently are described in scanty detail in research articles, which makes it difficult to interpret the results of many studies of VVA.

\section{Selection, Construction, and Scaling of Visual Stimuli}

The theoretical scheme given earlier requires the employment of a very wide variety of visual stimuli. This is for two reasons. First, the nature of the problem frequently dictates the type of visual stimuli that are employed in the investigation. For example, if one is investigating cognitive and emotional associations, copies of a wide variety of paintings would be useful. Second, many of the independent variables concern the characteristics of the stimuli. To investigate the effects of physical complexity, for example, one must construct stimuli that vary systematically in that regard.

\section{Types of Stimuli Employed}

Stochastically formed displays. In some cases the visual stimuli are constructed on the basis of probability models. This is the case for 
randomly constructed polygons, dot patterns, and checkerboard patterns. Such displays are constructed by a set of statistical rules rather than by an intentional effort to depict representational objects. A simple example is that of manipulating the "grain" of checkerboard patterns. All of the patterns occupy the same amount of space, e.g., 10 inches $X 10$ inches. The grain of the figure can be manipulated by the number of blocks into which the square is divided, varying from only four blocks up to a very large number. Specified percentages of blocks can be filled in either randomly or with some probabilistic contingency restraints. An example is a coin being flipped to decide whether each block is left vacant or blackened. There are many types of stochastic models that can be employed to construct stimuli with respect to some of the purely physical properties mentioned in our theoretical scheme.

Drawings. For many purposes, it has been necessary to hire artists to construct figures specifically related to particular stimulus variables. An example is in our studies of information conflict. We specified for the artist the cardinal features that an object should have at each level of information conflict (novelty), and he drew versions of the stimuli until they met our satisfaction. In the study of pleasantness it was necessary to have a series of outline drawings on a 5step continuum composed of female faces varying from very ugly faces to very pretty faces. (Of course all such sets of stimuli necessarily were subjected subsequently to psycho-physical scaling studies prior to their employment in studies of VVA.)

There are advantages and disadvantages of employing outline drawings. One disadvantage is that they are time-consuming and relatively expensive. On some occasions it has been necessary for the artist to draw several versions of the same picture before it appeared suitable. The second disadvantage is that there are many kinds of stimuli that would be prohibitively complex and would require the use of colors.

The major advantage of working with outline drawings is that one can manipulate the parti- cular stimulus variable in question and attempt to hold constant possible confounding stimulus variables. For example, there is so little detail in some of the outline drawings that amount of detail is not likely to be important in VVA. Since they are all in black and white, obviously colors and distributions of colors would not have an influence. By having the pictures drawn, one can emphasize the particular feature being investigated, e.g., the novel component. Also, with drawings one can depict impossible things, as in pictures we employ in studies of information conflict.

Posed scenes. On a number of occasions we have photographed posed scenes, such as a parent spanking a child or a young man and woman embracing. These were used to investigate various affectual states. The advantage of posed scenes over drawings is that, obviously, they are much more lifelike and thus might have stronger effects than outline drawings. However, posed scenes have their problems also, particularly in controlling for unwanted and frequently unsuspected confounding stimulus characteristics.

Sample pictures from daily life. Studies of VVA have used many pictures of scenes from daily life, ones that either were photographed specifically to meet some purpose or cropped from magazines and other printed matter. We presently are investigating 38 categories of reallife scenes (with five pictures in each category). The categories were formed in terms of our hypotheses about some of the determinants of VVA mentioned earlier. In many cases we photographed these in our environment, while in other cases they were taken from magazines.

Works of art. Particularly for investigations into variables relating to aesthetics, it is useful to employ photographic reproductions of works of art. One can do this either by making slides for a very broad "sample" of paintings or by collecting slides with respect to specified categories of paintings which are intended to represent one or more of the theoretical constructs mentioned previously. 


\section{Psychometric Requirements}

Only persons who have one foot in psychometric theory and one foot in experimental psychology can appreciate the demands that these two sub-disciplines place upon one another. What sounds perfectly logical from one perspective proves to be patently impractical from the standpoint of the other, and vice versa. Some of the salient principles whereby these two subdisciplines have been wedded in our research are as follows:

Multiple levels. Theories concerning VVA speak of various aspects of looking behavior as being a function of certian treatment conditions or characteristics of stimulus variables. The pioneering work in this area (e.g., Berlyne, 1960), primarily involved showing pictures that represented only two levels of the variable in question. For example, a comparison would be made of the amount of time spent looking at the picture of an ordinary house and a novel drawing of a house. In other instances, only several levels of a stimulus variable were investigated, such as several levels of complexity.

Another problem relating to number of levels concerns the range of stimuli employed in the investigations. When we started investigating the physical complexity of random polygons, it was not uncommon to see reports in journal articles in which the highest level represented was 40 random sides. Now we are working with random polygons that have up to 200 sides. A major effort of our project has been to develop sets of stimuli that varied widely and at numerous points on the continuum of the particular stimulus characteristic being investigated. This permits us to make firmer, more general statements about the nature of functional relationships.

Scaling data. For the development of stimulus scales, we relied on two types of data. First, in some instances we could rely on the physical properties of the stimuli. One example is with our random polygons, in which complexity could be measured in terms of the number of random sides used to generate the figure.
Another example was in the number of different encodable stimuli in a display, which was indexed quite simply in terms of the proportions of elements of different kinds that were shown. Such physical properties served directly to develop interval or ratio scales.

With most of the stimuli that we have employed, it is logically impossible to use as scaling data any measurable physical properties. This is illustrated with our drawings of incongruous juxtapositions of animal parts with other elements such as an outline drawing of a cow with symmetrical polka dots. Actually, in information-theoretic terms, the splotches on an ordinary cow would represent a higher level of complexity than would the polka dots, but, of course, the polka-dotted cow is responded to as being much more novel. In the outline drawings that we employ to depict various motivational states, e.g., a child receiving a spanking, there is nothing that can be measured physically by ruler, photo-electric cell, or any other physical process that would index the variable in question. The only way to index novelty, motivational states, associations, and most of the important stimulus variables is in terms of subjects' reactions. Consequently, a large part of our research effort has been dedicated to gathering responses to our stimuli which then could be employed for psychophysical scaling. These scales were subsequently used in studies of VVA. For this purpose we obtained subject reactions of many kinds. We relied heavily on rating scales, e.g., for the measurement of pleasantness. Free associations were obtained to scale stimuli in terms of some of the variables relating to meaning-processing. In our studies of incongruous drawings, children were asked to rank-order the pictures within each set from the most usual to the most unusual. In these and other ways, it not only was practicable to employ subjects' reactions as data for scaling stimuli, but logically there was no other type of data that could possibly have served that purpose.

Need for replicate stimulus sets. In the investigation of any variable in our conceptual scheme, it was necessary to employ more than 
one set of materials in each study. In a study of effects of sets of incongruous drawings on amount of looking time, we typically would employ four or more different sets, each of which involved four or more levels of novelty. Similarly, in investigating effects of different levels of com. plexity as evidenced in randomly constructed geometrical forms, we typically employed four or more sets of stimuli that varied on as many as six levels.

The reason for employing replicate sets of this kind is to give the overall results a form of content generality, much as is traditionally involved in the construction of psychological tests. We could not trust the results that we obtained from one set alone, simply because there may have been something peculiar about the picture used to represent a particular level or even about a whole set of pictures. Looking through the literature on VVA over the last ten or so years, one can find authors making much out of a particular dip in a curve or a final downturn; but frequently these curves are based on one set of stimuli only. One should employ multiple sets of stimuli for any variable, and these should be scaled psychophysically before studies of VVA are undertaken.

Need for minimal overlap. Our investigations require levels of stimuli that barely overlap in terms of scale properties. A cardinal example is in respect to information conflict. We can not employ such scales unless nearly all subjects agree on the way on which stimuli should be rank-ordered with respect to novelty. Similarly, in our scales that are constructed in terms of physical principles, it is necessary to employ stimuli at each level (e.g., of complexity) that do not overlap with other levels. Without these clean-cut differences between stimuli at adjacent levels on each scale, we could not expect to obtain clear results in VVA.

Floor and ceiling effect. In developing stimuli, we frequently encounter natural floors in which either one is working with a logical zero point or one can not obtain a lower level on the stimulus continuum in scales based on subjects' ratings. One can not obtain a polygon with fewer than three sides or a collection of objects less heterogeneous than one in which all the objects are alike. Similarly, in our scales that are obtained primarily from the pretesting of subjects' reactions to pictures rather than in terms of physical properties, we frequently found floor effects or logical zero points. For example, we can not draw a picture of a cow that will be rated clearly as more usual than the picture of the cow that we presently employ in that set. Similarly, in many efforts we have been unable to construct a more bizarre concatanation of cow, elephant, and airplane parts than the most incongruous stimulus in that series. Logically, then, we have covered the range from the bottom to the top, which leaves little argument about what would happen if stimuli were more extreme in either direction. Some of our sets constructed in terms of physical properties have been made so complex or so extreme in terms of some other physical property, that it is doubtful that any practicable increases in scale values would result in different findings regarding VVA.

Scaling techniques. We have found it necessary to rely on only some very simple approaches to scaling. For example, we have encountered no case in which multidimensional scaling would have been useful. Such scaling methods are used when stimuli are expected to differ from one another prominently on more than one dimension and/or the cardinal dimensions can not be specified in advance. Neither has been the case in our investigations. Rather, we have worked from a hypothesis-testing model, in which our effort is to measure each theoretical variable separately, e.g., information conflict or complexity. We make efforts to purify our stimulus materials of any other psychological dimensions that might intrude themselves. Also, the employment of replicate sets as mentioned above helps to average out any confounding stimulus variables that might by chance be represented in one of the sets but not in others.

As mentioned above, some of our unidimensional scales were developed directly from their physical properties, such as levels of complexity in geometrical forms and relative homogeneity of elements of visual displays. The numerical 
characteristics of the displays themselves provide sensible scales, although in some circumstances we found that the overall nexus of lawful relations among independent variables and with dependent variables was simplified by systematic modifications of the intervals, e.g., we found it mathematically convenient to work with logarithmic values concerning complexity in random polygons.

For our scales based on data obtained from human impressions, it was not necessary to apply any complex scaling techniques. All of the scaling methods that have grown out of Thurstone's law of comparative judgment did not apply, because the research required nearly nonoverlapping distributions of individual differences. Consequently. where stimuli were based on ranking we simply used the average ranks over subjects as though they constituted an interval scale. With scales based on ratings, e.g., 9-step scales concerning judged pleasantness of pictures, we simply took the average ratings to form interval scales.

\section{Measurement of Complexity- A Psychometric Case History}

One of the constructs in our theoretical scheme for explaining VVA is physical complexity, which we have investigated extensively (see Wilson, 1973; Wilson and Nunnally, 1973). Although many types of geometrical configurations have been employed to investigate effects of complexity on VVA (e.g., random dot patterns), randomly generated polygons have been used most frequently. Such polygons usually are described in terms of number of randomly constructed sides. A technique for generating such polygons was developed by Attneave and Arnoult (1956), which we employed in some of our earlier investigations. However, this method proves quite awkward when generating polygons that have more than about 40 sides. When we reported findings in the literature relating degree of complexity in random polygons and various aspects of VVA, our critics frequently rejoined that the reported relationship would have changed in appearance if higher levels of com- plexity had been employed, i.e. the typical in creasing monotonic relationship from threesided up to about forty-sided figures. To meet this challenge we developed a semi-computerized procedure for developing immensely complex random polygons. The method is described in detail in Wilson and Nunnally (1973). The major steps in the procedure are as follows. First, a computer is programmed to print consecutive numbers at random locations in a specified areas, e.g., one foot square. Second, by hand, one connects all of the outside points, thus producing a figure that includes all random points within it. Next, one randomly selects pairs of adjacent points on this outside figure and creates a triangle by randomly attaching these two points to one of the points within the figure. This process is continued through various cycles until the desired number of random sides are constructed (the actual details are more complex than this but straight-forward in practice).

An example of a 200-sided polygon is shown in Figure 1. The only practical limit to the number of sides that one can produce in a polygon by this method is the fact that in extremely complex forms some of the sides developed by this method are so small as to not be visually discernable. This method allows us and others to em-

Figure 1

A 200-Sided Randomly Generated Polygon (from Wilson and Nunnally, 1973)

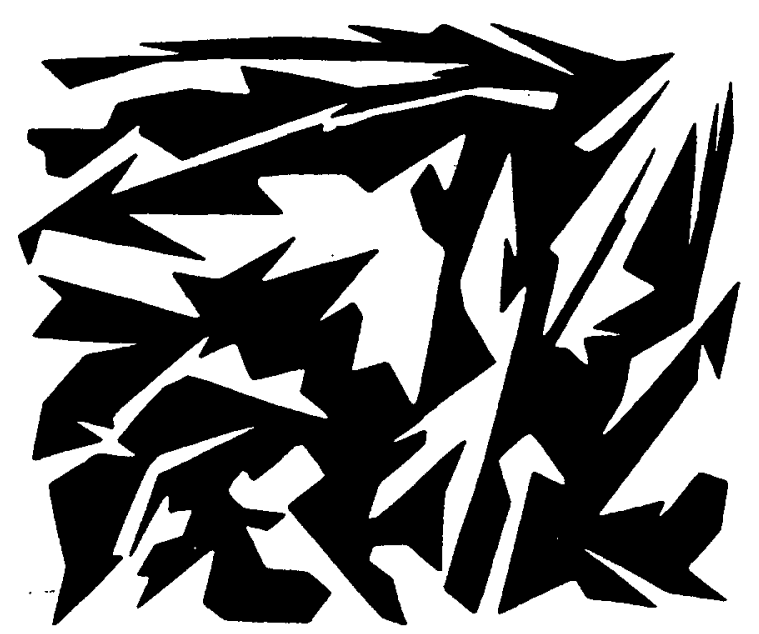



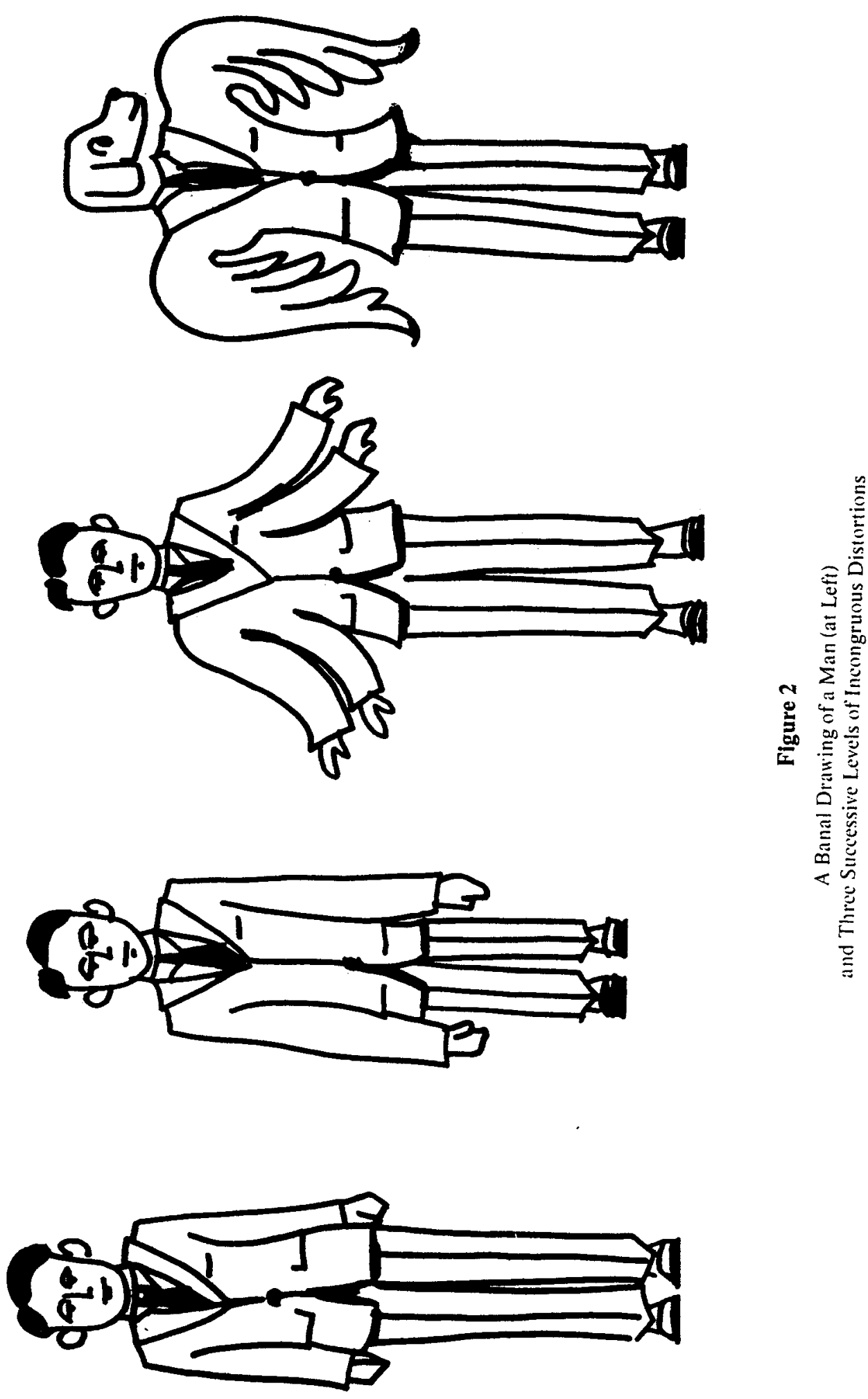
ploy scales of physical complexity in random polygons to any level that is needed to fully explore effects on VVA or other psychological processes.

\section{Illustrative Findings}

Space permits only the summarizing of some of the most pervasive and conclusive findings from our investigations of VVA. More extensive summaries and numerous references to particular experiments are given in Lemond (1973), Nunnally (1976), Nunnally and Lemond (1973), and Wilson (1973).

Information conflict. There is a monotonically increasing relationship between degree of information conflict (an important type of novelty) and amount of VVA. A typical set of pictures used in such investigations is shown in Figure 2. A typical set of results is shown in Figure 3.

Figure 3

Relationship Between Levels of Novelty and Average Viewing Time (from Faw and Nunnally, 1968a)

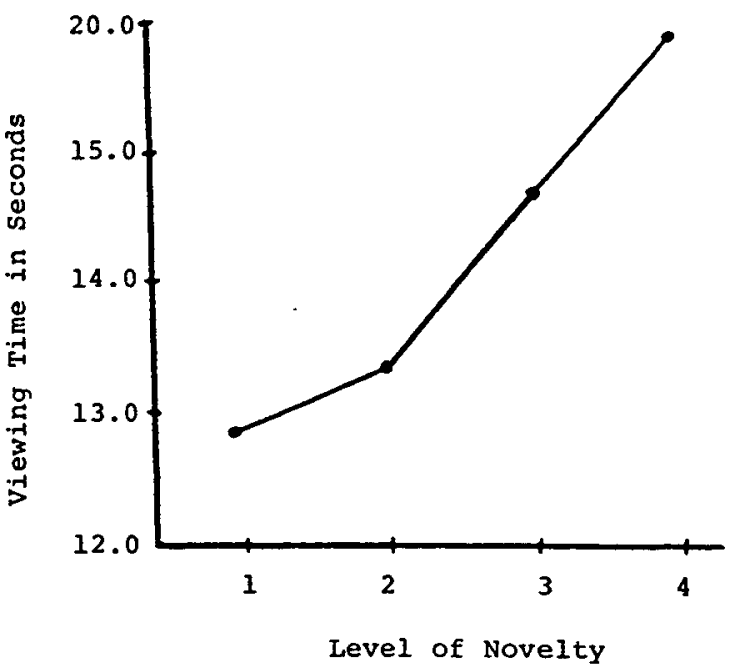

Representational elements. There is a monotonically increasing relationship between the number of different representational elements (thing-like objects) in visual displays and amount of VVA (evidence summarized by Wilson, 1973).
Physical complexity. There is a monotonically increasing relationship between physical complexity (e.g., as manifested in number of random sides of a polygon or number of random dots in a pattern) and amount of VVA. The measurement of physical complexity in geometrical forms was discussed previously; a typical set of experimental results is shown in Figure 4. The levels of complexity shown there are $3,10,20,40$, and 80 random sides expressed in terms of $\log _{10}$. We have unpublished data showing that the monotonically increasing trend continues up to 200 random sides.

Relationship Between Levels of Complexity of Random Polygons and Average Viewing Time (from Nunnally and Lemond, 1973)

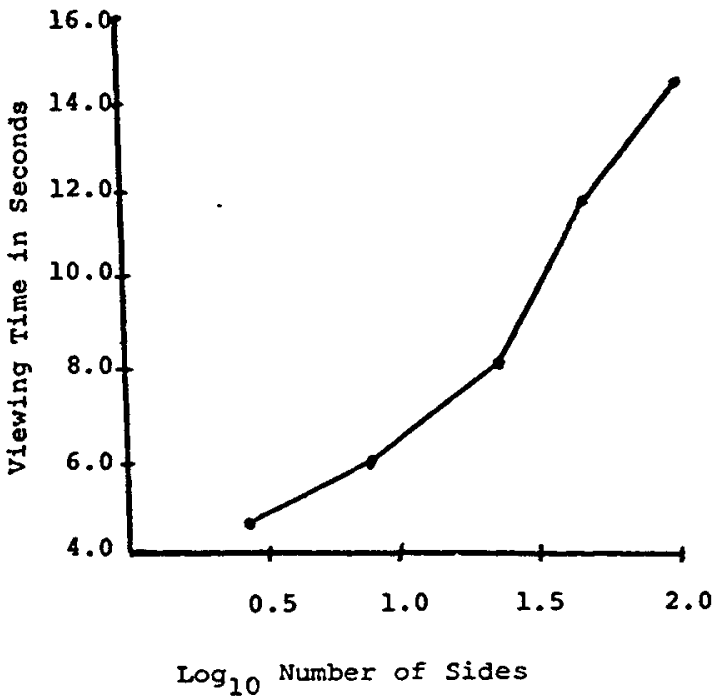

Pleasantness. There is no general relationship between subjects' ratings of pleasantness of stimuli and amount of VVA. Findings with one type of stimuli do not hold with other types of stimuli, e.g., rated pleasantness of female faces versus outline drawings of scenes showing various types of social interactions. There has been a tendency throughout our studies (by no means entirely consistent) for pleasant pictures to dominate neutral pictures, but VVA with respect to various levels of negative affect evidenced in ratings is unpredictable. There is 
STUDIES OF VOLUNTARY VISUAL ATTENTION-

some evidence from our studies (e.g., Faw and Nunnally, 1971) that people compulsively stare at some types of unpleasant pictures, particularly those concerning any type of human harm.

Familiarization. With various experimental approaches, numerous studies have been performed on the effects of prior familiarization of stimuli on subsequent VVA. It is quite conclusive that familiarization reduces subsequent VVA, both when stimuli are subsequently viewed separately or when a familiarized stimulus is paired in the same display with an unfamiliarized stimulus. However, there are many puzzling features regarding these investigations which do not fit any simple generalization beyond the observed major effect, e.g., our studies show no effects of such prior familiarization on subsequent ratings of pleasantness, and the effects of amount of familiarization in our studies level off after only a few seconds rather than increase monotonically.

Other findings. Many findings by us and others on numerous experimental treatments are referenced in the summary articles mentioned above. These include effects of various physical characteristics of stimuli, of amounts and kinds of sensory isolation, of instructional sets, and of interposing various types of vigilance tasks between trials of VVA. Some of the findings from these investigations have been clearcut, but they have not been replicated or studied as broadly as those in the five categories discussed above.

\section{An Ilustrative Set of Relationships}

Table 1 shows the results of a principal-components factor analysis of a set of intercorrelations of numerous variables in our theoretical scheme and VVA. The first variable is viewing time obtained from a group of 36 college students, which as usual we have found convenient to express in terms of $\log _{10}$ of seconds. VVA consisted of instrumental viewing responses employing a Carousel slide projector in the manner discussed previously. A disguised set of instruc-
Table 1

Principal Components Factor Loadings for VVA (Variable 1), Physical Complexity (Variable 2), and Measures of Eight Variables that are Theoretically Iniked to VVA

\begin{tabular}{lllll}
\hline & & & \\
& & II & $h^{2}$ \\
\hline 1. $\log _{10}$ Viewing Time & .93 & .03 & .87 \\
2. $\log _{2}$ Number of Sides & .96 & -.10 & .93 \\
3. Number of Things Seen & .83 & -.27 & .77 \\
4. Part-Whole Response & -.86 & .09 & .75 \\
5. Rated Novelty & .99 & -.01 & .99 \\
6. Rated Complexity & .98 & -.10 & .97 \\
7. Rated Interestingness & .97 & .18 & .98 \\
8. Confidence of Naming & -.87 & .06 & .76 \\
9. Prob. of Occurrence & .99 & .02 & .98 \\
10. Rated Pleasantness & .37 & .92 & .99 \\
\hline
\end{tabular}

tions was used in which subjects were told that the visual stimuli were being made available simply as an incidental part of a study of auditory thresholds. The second variable is that of number of random sides of polygons, which we expressed as $\log _{2}$ to fit information-theoretic concepts. There were six levels of complexity, involving random sides of $3,10,20,80$, and 200 respectively. The third variable concerns ratings by subjects of the number of things that they could see (representational elements) in each geometrical form, which was rated on a scale from 1 to 10 . With the fourth variable, subjects rated whether they mainly were responding to one part of the geometrical form or to the whole form in supplying a name for each stimulus (whole was scored 1 and part was scored 0 ). The remaining six variables were rated on a 9-point continuum with 9 representing highly novel, complex, interesting, confidence in giving a name, and the improbability of encountering something resembling the geometrical form in real life. Variable number 10 involved pleasantness of the geometrical form. Data for variables 3 through 10 were obtained from the responses of 94 undergraduate students at Vanderbilt.

The full set of intercorrelations and numerous other statistics relating to this investigation are yet to be published. However, for current pur- 
poses, the important results are summarized in Table 1. Unities were placed in the diagonal of the correlation matrix before factoring, and no rotations were necessary for the factors. The first factor explained nearly all of the total variance. Factor 2 is almost entirely a specific factor relating to rated pleasantness. Two principles are very obvious here. First, rated pleasantness has a very low relationship with VVA or any of the other variables in the study. (In studying other types of materials we have even found some significant negative correlations between rated pleasantness and VVA). Second, all of the other variables correlate highly with VVA; and the general factor explains most of the total variance. Some important findings are: (1) as we hypothesized, "number of things seen" correlates highly both with physical complexity and VVA; (2) confidence in giving a name has an inverse relationship with VVA; and (3) the more complex the figure, the more the naming response tends to be related to a subpart of the total figure rather than the figure as a whole.

Two points remain to be made. The very high correlations of our variables with looking time and with one another is both bad and good. It is always encouraging to find extremely high relationships with one's major dependent variable, VVA in this case, but the extremely high correlations among the independent variables also poses a problem for us. One way of looking at it is that in such randomly generated geometrical forms, constructs that we intend to study separately are "inherently confounded," which has forced us to construct special sets of materials relating to complexity, novelty, and other variables in which the separate effects of these constructs could be investigated (e.g., as in the study reported by Lemond, 1973). Second, this set of results pertains to randomly constructed polygons (and we hypothesize to other classes of stimuli that have been used to date to investigate complexity), but very different results might be found with other classes of stimuli, such as those that we use to investigate information conflict, number of representational elements, and all of the motivational variables.

\section{Psychometric Challenges}

The first author has long maintained that the slow progress in the development of psychology as a science has been closely related to the slowness of developing adequate measurement methods for the salient constructs in theories. Psychology is overpopulated with fascinating theories that never quite touch ground because the major terms are not rooted in concrete measurement operations, and this has been the case in the study of VVA. Most of the studies have been simple demonstrations (e.g., people will look longer at a novel stimulus than a non-novel stimulus), and for such crude beginnings one does not need multi-point scales, replicate sets of materials, and the other refinements required for a careful investigation of functional relationships among and between independent and dependent variables.

One can look back at the major constructs mentioned in the theoretical scheme presented earlier in this paper to see the many measurement issues that will be involved in fully investigating the major determinants of VVA. For example, how does one measure specific emotions, degree of information conflict, arousal, and the other constructs in the scheme? Actually, it is not difficult at all to think of plausible approaches to measuring these salient variables. The psychometric techniques mostly are already available, and they grow out of the general psychometric theory coming from classical psychophysics, mental test theory, multivariate analysis, and modern statistics.

Some of the variables that can be measured in terms of direct physical properties simply have not been sufficiently exploited. The variable of arousal is a good example. The author and his colleagues (Nunnally, Knott, Duchnowski, and Parker, 1967) found that pupillary response is an excellent measure of overall arousal, and this can be measured simultaneously with the measurement of amounts and kinds of VVA. This makes the problem of equipment usage and related technical details more complex, but it is entirely feasible. Both the cognitive and motiva- 
tional components of association can be investigated, the former somewhat easier than the latter, by methods of free association and other techniques. Such is the case with the other constructs mentioned in the theoretical scheme: it takes no great stretch of the imagination to think of plausible inroads for their measurement of subsequent use in experiments on attention.

Much of our lives are spent in visually scanning the human and material environment, and the relative amounts of time spent in looking at different things "out there" are far from random. Our investigations and those of others have shown that there are some solid principles relating measurable constructs to amounts and kinds of voluntary visual attention, which gives hope of resolving an even more precise and complete network of lawful relations in the future.

\section{References}

Attneave, F. \& Arnoult, M. D. The quantitative study of shape and pattern perception. Psychological Bulletin, 1956, 53, 452-471.

Berlyne, D. E. Conflict and information-theory variables as determinants of human perceptual curiosity. Joumal of Experimental Psychology, 1957, 53, 399-404.

Berlyne D. E. The influence of complexity and novelty in visual figures on orienting responses. Journal of Experimental Psychology, 1958, 55, 289. 296.

Berlyne, D. E. Conflict, arousal and curiosity. New York: McGraw-Hill, 1960.

Berlyne, D. E. Studies in the new experimental aesthetics. New York: Wiley, 1974.

Berlyne, D. E. Aesthetics and psychobiology. New York: Appleton-Century-Crofts, 1971.

Berlyne, D. E. Studies in the new experimental aesthetics. New York: Wiley, 1974.

Cantor, J. H., \& Cantor, G. N. Functions relating to children's observing behavior to amount and recency of stimulus familiarization. Joumal of $E_{x}$ perimental Psychology, 1966, 72, 859-863.

Dember, W. N. \& Earl, R. Analysis of exploratory, manipulatory, and curiosity behaviors. Psychological Review. 1957, 64, 91-96.

Durham, R. L., Nunnally, J. C., \& Lemond, L. C. The effects of levels of information conflict on visual selection. Perception \& Psychophysics, 1971, 10. 93-96.

Faw, T. T. \& Nunnally, J. C. The effects on eye movements of complexity, novelty and affective tone.
Perception and Psychophysics, 1967, 2, 263-267.

Faw, T. T. \& Nunnally, J. C. The influence of stimulus complexity, novelty, and affective value on children's visual fixations. Journal of Experimental Child Psychology, 1968, 6, 141-153. (a).

Faw T. T., \& Nunnally, J. C. A new methodology and finding relating to visual stimulus selection in children. Psychonomic Science, 1968, 12, 47-48 (b).

Faw, T. T. \& Nunnally, J. C. Effects of familiariza: tion with incongruous stimuli on their dominance in visual selection. Psychonomic Science, 1970, 19, 359-361.

Faw, T. T. \& Nunnally J. C. The influence of stimulus incongruity on the familiarity effect in visual selection. Perception and Psychophysics, 1971, 9, 150-154.

Fiske, D. W. \& Maddi, S. R. Functions of varied experience. Howewood, Illinois: Dorsey Press, 1961.

Fowler, H. Curiosity and exploratory behavior. New York: MacMillan, 1965.

Leckart, B. T. \& Bakan, P. Complexity judgments of photographs and looking time. Perceptual and Motor Skills, 1965, 2, 16-18.

Lemond, L. C. The influence of degrees of complexity, incongruity, and pre-exposure on the familiarity effect in visual selection. Doctoral dissertation, Vanderbilt University, 1973.

Nunnally, J. C. A human tropism. In S. R. Brown \& D. J. Brenner (Eds.), Science, psychology, and communication: Essays honoring William Stephenson. New York: Teachers College Press, 1972, 255-277.

Nunnally, J. C. Meaning-processing and rated pleasantness-Effects on aesthetic preference. Scientific Aesthetics, in press, 1976.

Nunnally, J. C., Duchnowski, A. J., \& Knott, P. D. Association of neutral objects with rewards: Effects of massed versus distributed practice, delay of testing, age, and sex. Journal of Experimental Child Psychology. 1967, 5, 152-163.

Nunnally, J. C., Knott, P. D., Duchnowski, A. J., \& Parker, R. Pupillary response as a general measure of activation. Perception and Psychophysics, 1967, 2. 149-155.

Nunnally, J. C. \& Lemond, L. C. Exploratory behavior and human development. In H. W. Reese (Ed.) Advances in child development and behavior (Vol. 8). New York: Academic Press, 1973.

Wilson, W. H. The influence of stimulus complexity on exploratory behavior: Implications for a meaning-processing model. Doctoral dissertation, Vanderbilt University, 1973.

Wilson, W. H. \& Nunnally, J. C. A technique for producing highly complex visual stimuli. Behavior Research Methods and Instrumentation. 1973, 5. 266.268. 


\section{Acknowledgements}

Preparation of this article was supported in part by a research grant to Jum $\mathrm{C}$. Nunnally from the $\mathrm{Na}$ tional Institute of Child Health and Human Development (Number HD MH-09337-02).

\section{Author's Address}

Jum C. Nunnally, Department of Psychology, Vanderbilt University, 134 Wesley Hall, Nashville TN 37240 . 